\title{
Theory of Pulsational Instabilities of Hot Stars
}

\author{
Alexei A.Pamyatnykh \\ Copernicus Astronomical Center, Bartycka 18, 00-716 Warsaw, Poland \\ Institute of Astronomy, Pyatnitskaya 48, 109017 Moscow, Russia
}

\begin{abstract}
Basic physical mechanism of the pulsational instability in OB stars is shortly reviewed. Similarities and differences in the driving effect acting in these objects and in the classical pulsating variables are emphasized. Updated theoretical instability domains in the H-R diagram, in the $\log g-\log T_{\text {eff }}$ and in the Period - $\log T_{\text {eff }}$ diagrams are presented and compared with the observational data on $\beta$ Cephei, SPB and some other types of hot variables.
\end{abstract}

\section{The opacity mechanism in Main Sequence stars}

This short review is devoted to the new instability strip in the HR diagram which was determined theoretically approximately five years ago (see Gautschy \& Saio 1996 and references therein). It is located along upper part of the Main Sequence and is populated by Slowly Pulsating B-type (SPB) stars, $\beta$ Cephei stars and other types of $O B$ variables. In its uppermost part, at luminosities of about $10^{6}$ of the solar value, the OB-star instability strip extends well beyond the Main Sequence and probably merges with the instability strip of classical Cepheids. At these high luminosities both radial and nonradial oscillations correspond mostly to unstable strange modes which are discussed by W. Glatzel in these Proceedings.

In the upper Main Sequence, the pulsational instability is caused essentially by the same opacity (or $\kappa^{-}$) mechanism which drives pulsations in the classical instability strip. An exception is that in OB stars this mechanism is connected with a different opacity maximum. Figure 1 illustrates the opacity behaviour at astrophysical conditions. The main local maxima (ridges or bumps) are due hydrogen ionization, second helium ionization and due to absorption by excited ions of metals. In various stellar models these three bumps occur at approximately the same temperatures $\left(1.0-1.2 \times 10^{4} \mathrm{~K}\right.$, $4.5-5.0 \times 10^{4} \mathrm{~K}$ and $1.5-2.0 \times 10^{5} \mathrm{~K}$, respectively), but at different densities and geometrical depths. The third maximum was missing in the earlier data, it has been recognized recently by taking into account a huge number of absorption lines produced by intrashell transitions in iron-group elements (for newest versions of two independent series of the opacity tables, OPAL and OP, see Iglesias \& Rogers 1996 and Seaton 1996, respectively). In regions around opacity maxima the radiative flux from the interiors may be partly blocked during compression and converted into kinetic energy of oscillations. 
It can be seen from Fig. 1 that all stellar models cross the opacity bridges. However, not all of the models are pulsationally unstable and only certain kinds of pulsation are excited in different models.

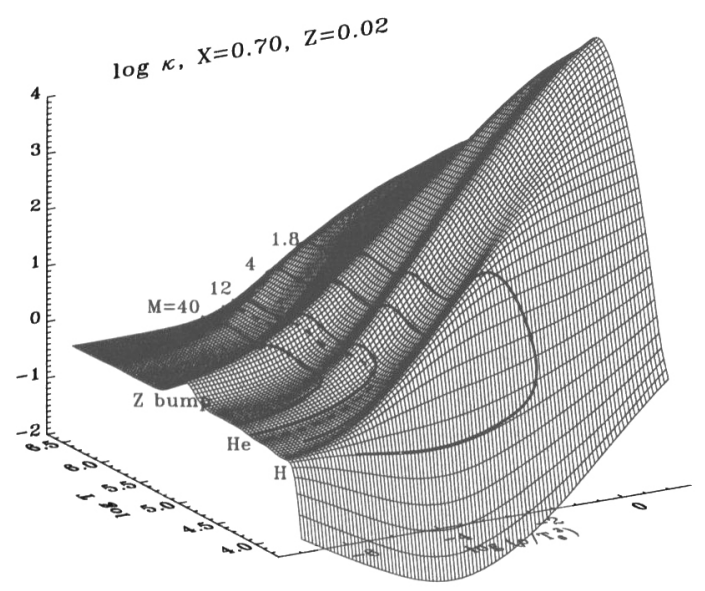

Fig. 1. Stellar opacity versus $\log T$ and $\log \rho / T_{6}^{3}$ where $T_{6} \equiv T / 10^{6}$. An OPAL table from Iglesias \& Rogers (1996) for a standard chemical composition was used. Thick solid lines correspond to the ZAMS models of $1.8-40 M_{\odot}$, dashed line corresponds to the $12 M_{\odot}$ model on the TAMS (Terminal-Age Main Sequence). Hydrogen, helium and metal $(Z)$ ridges are marked by corresponding symbols.

To explain the opacity mechanism, it is useful to consider contributions from different layers in stellar interiors to the thermodynamic work integral, $W$, which is the net energy gained by an oscillation mode during one cycle of pulsation (for detailed discussion, see Dziembowski (1995) and Gautschy \& Saio 1995 and references therein). It is easy to show that a region in the stellar envelope in which the opacity derivative $\kappa_{T}$ (more exactly, $\kappa_{T}+$ $\left.\kappa_{\rho} /\left(\Gamma_{3}-1\right)\right)$ is increasing outwards, will contribute to the driving. Here $\kappa_{T}=(\partial \ln \kappa / \partial \ln T)_{\rho}, \kappa_{\rho}=(\partial \ln \kappa / \partial \ln \rho)_{T}$, and $\Gamma_{3}-1=(d \ln T / d \ln \rho)_{\text {ad }}$. If opposite is true, the region will contribute to the damping the oscillations. Note that spatial variation of $\left(\Gamma_{3}-1\right)$ due to ionization of abundant elements or due to radiative pressure effects near opacity maxima has only small influence on OB star instability.

Two additional conditions must be fulfilled to excite global stellar pulsation in a given mode: (i) the amplitude of oscillation must be relatively large and slowly varying in the potentially driving region, (ii) thermal timescale 
in the driving zone, $\tau_{\text {th }}(r)=\int_{r}^{R} T c_{P} d M / L$, must by comparable or longer than the oscillation period. Otherwise, potentially driving region remains in thermal equilibrium during pulsation (neutral stability). The last condition means that to excite the oscillations, the opacity bump has to be located at an optimal geometrical depth in the stellar envelope.

The efficiency of the $\kappa$-mechanism in representative models of $\beta$ Cephei, SPB and $\delta$ Scuti variables is demonstrated in Fig. 2. The stars are located in the middle of corresponding instability domains in the HR diagram. It is easily to see that main driving in all models takes a place in the layers with steep gradient of the opacity derivative near relevant opacity bump. Acoustic
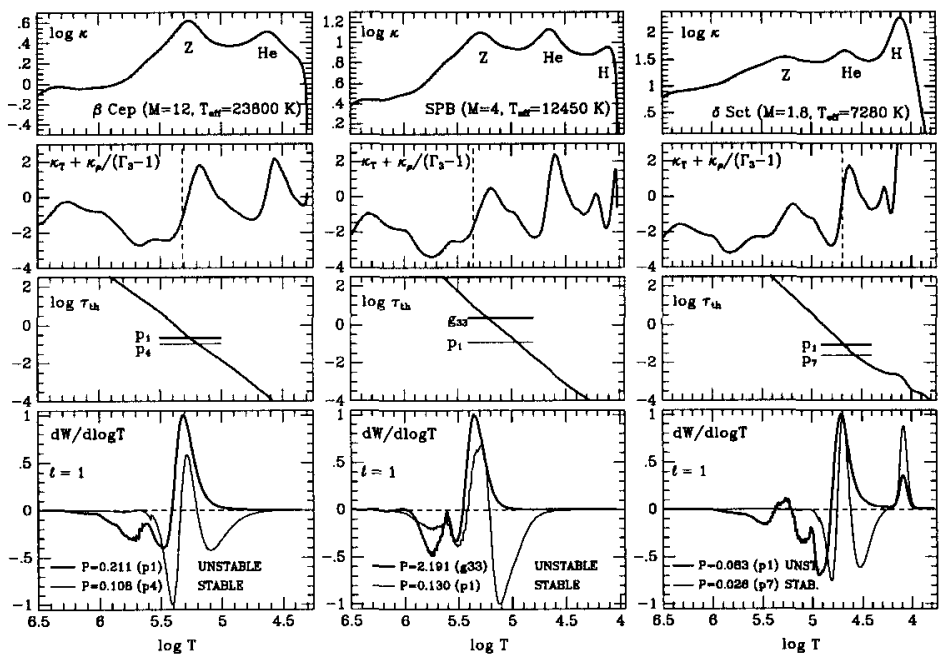

Fig. 2. Opacity, $\kappa$, opacity derivative, $\kappa_{T}+\kappa_{\rho} /\left(\Gamma_{3}-1\right)$, thermal timescale, $\tau_{\text {th }}$ (in days), and differential work integral, $d W / d \log T$ (arbitrary units, positive in driving zones), for selected pulsation modes $(\ell=1)$, plotted versus temperature for three representative models of $\beta$ Cephei (left), SPB (middle) and $\delta$ Scuti (right) variables. All models have initial chemical composition $X=0.70$ and $Z=0.02$. Dashed vertical lines mark the position of the maximum driving for the unstable modes shown in the lower panels. Horizontal lines in the $\tau_{\mathrm{th}}$ diagrams correspond to the periods of selected modes.

mode $p_{1}$ (as well as other low-order radial and nonradial modes) is excited in $\beta$ Cep and in $\delta$ Sct model, but it is damped in SPB model. The helium bump region, where pulsations of $\delta$ Sct stars and other classical variables are driven, is neutrally stable in B stars due to very short thermal scale there. However, the timescale requirement can be satisfied at the position of the 
metal bump. In the $\beta$ Cep model of $12 M_{\odot}$, the thermal timescale there is comparable with periods of the low order p-modes. In the SPB model of $4 M_{\odot}$, the thermal timescale at the metal bump is approximately 20 times longer because the bump is located deeper and due to smaller luminosity of the star. It is comparable now with periods of the high-order g-modes which become unstable. For low order g- or p-modes the damping between helium and metal bump is activated, therefore these modes are stabilized. More detailed discussion of the SPB versus $\beta$ Cep-type instability in terms of the work integral is given by Dziembowski et al. (1993) and Dziembowski (1995).

The long-period, high-order g-mode instability extends to high luminosities, well beyond the observed SPB domain in the HR diagram (see Pamyatnykh 1998 and Fig. 4 below). In this region, two separate frequency ranges appear in the oscillation spectrum, which can be easily understood with help of Fig. 3. In upper panels, the MS evolution of the spectrum of quadrupole $(\ell=2)$ modes for a $30 M_{\odot}$ star is shown. In a model of $\log T_{\text {eff }} \approx 4.44$ both high-order $g_{14}-g_{19}$ modes with periods $2.4-3.1$ days and low-order $p_{0}$, $g_{3}-g_{6}$ modes with periods $0.6-1$ days are excited by the metal opacity bump. (We classify acoustic and gravity modes according to distribution of their kinetic energy inside the star.) For these modes the oscillation amplitude is large only in the outer layers where driving occurs. In deep interiors, the amplitude behaviour for these two groups of modes is very different but the contribution to work integral is negligible in both cases. On contrary, intermediate modes which have relatively small amplitudes in the metal bump region, are stabilized in deep layers. A small driving at $r / R \approx 0.55$ caused by the local opacity maximum at $\log T \approx 6.25$ is not enough to outweigh the damping. Note that in hotter models, the high-order g-modes may also have large amplitudes in the potentially driving zone, but the thermal timescale is short there in comparison with the periods.

\section{Instability domains}

Figure 4 shows the instability domains along the upper Main Sequence. The models were computed using OPAL opacities and a standard stellar evolution code developed by B. Paczyński, M. Kozłowski and R. Sienkiewicz (private communication). The linear nonadiabatic analysis of low-degree oscillations $(\ell \leq 2)$ was performed with a code developed by Dziembowski (1977). For general consideration, the effects of stellar rotation, mass loss and convective overshooting were neglected.

Slowly Pulsating B star models are unstable only to nonradial high-order gravity modes. Due to very dense frequency spectrum, a lot of modes are unstable simultaneously: for example, in the TAMS $4 M_{\odot}$ model, the modes $g_{43}-g_{85}$ of $\ell=1$ and $g_{36}-g_{81}$ of $\ell=2$ are excited (the period ranges are 2.0-4.5 and 1.4-2.7 days, respectively). As it was discussed in the previous 

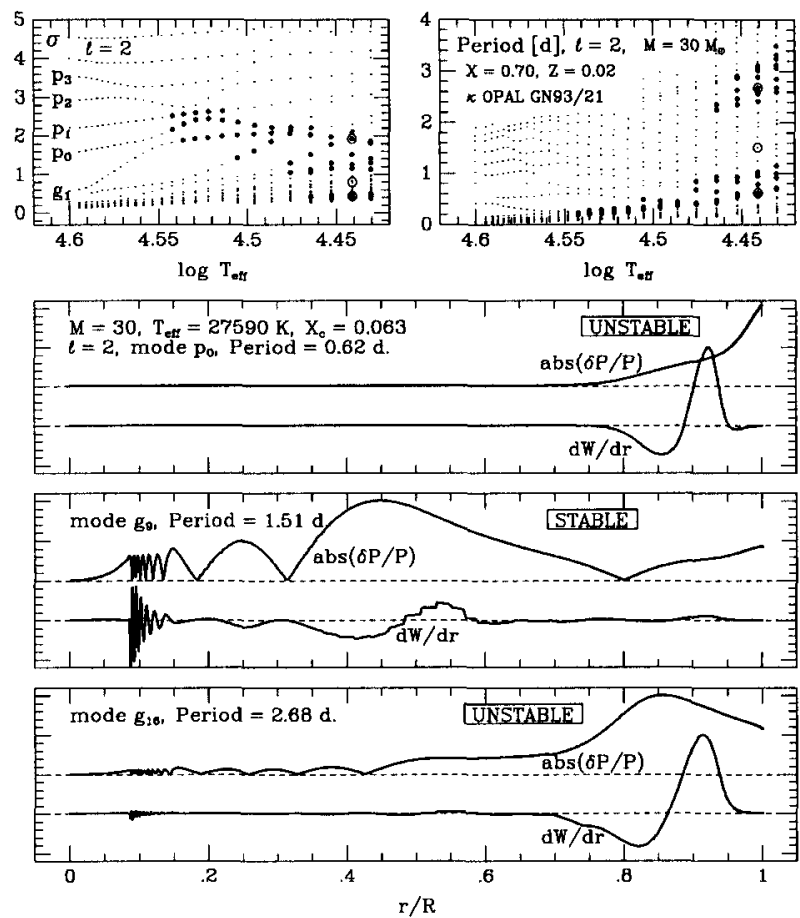

Fig. 3. Upper panels: Dimensionless frequencies, $\sigma=\omega / \sqrt{4 \pi G\langle\rho\rangle}$, and periods of quadrupole modes for a $30 M_{\odot}$ star in its MS evolution (from the left to the right). The small and large dots correspond to stable and unstable modes, respectively. Open circles at $\log T_{\text {eff }} \approx 4.44$ mark modes shown in the lower panels.

Lower panels: The differential work integral, $d W / d r$, and the pressure eigenfunction, $\delta P / P$ (both in arbitrary units), for three selected modes. Zero-lines for the variables are shifted one relative to another. The metal opacity bump is located at $r / R \approx 0.916$.

section, this instability extends to high luminosities, the extension is continuous for the OP opacities (Pamyatnykh 1998) or for models with convective overshooting resulting in a widening of the MS band. For the SPB domain and its extension, the computed Red Edge coincides with the TAMS, which is explained by very strong damping of the high-order gravity modes in evolved post-MS star models.

In fact, the opacity driven domains in the HR diagram present a sequence of several instability strips shifted in effective temperature one after another, as it can be easily understood from the position of blue (hot-temperature) boundaries. From the right to the left, we have classical instability strip driven by helium bump, high g-mode instability strip, and $\beta$ Cep instability 

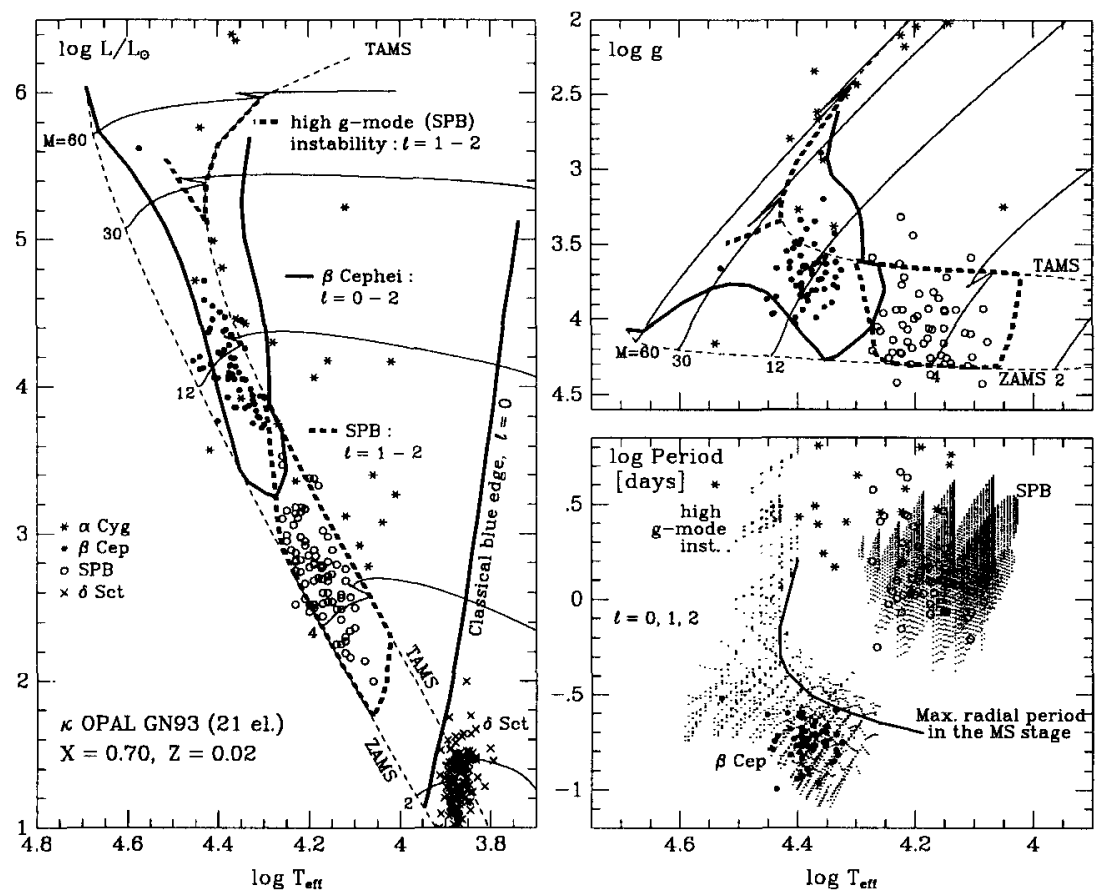

Fig. 4. Left: Instability domains in the upper Main Sequence. For the classical instability strip, only blue edge is shown. Thin solid lines show evolutionary tracks for a few stellar models with masses given in solar units. Thin dashed lines show ZAMS and TAMS. The positions of photometrically variable stars are due to L. A. Balona (private communication on $\beta$ Cep in open clusters), Sterken \& Jerzykiewicz (1993, $\beta$ Cep), North \& Paltani (1994, SPB), Waelkens et al. (1998, $\alpha$ Cyg and SPB), Garcia et al. (1995, $\delta$ Sct).

Right top: $\beta$ Cep and high-order g-mode (SPB-type) instability domains in the the $\log g-\log T_{\text {eff }}$ diagram.

Right bottom: Periods of unstable low-degree modes in $\beta$ Cep and SPB domains. For clarity, only MS models of $M \leq 40 M_{\odot}$ are considered in the $\beta$ Cep domain (post-MS models result in a decrease of the gap between $\beta$ Cep and SPB domains). Solid line marks the position of the TAMS line for radial pulsations in the $\beta$ Cep domain. All points to the right of the line correspond to low-order nonradial modes in this domain. Only main observational periods are plotted for multiperiodic variables. 
strip, both driven by $Z$ bump. Different slope of the SPB and especially of the $\beta$ Cep domain is caused partly by effects of large radiative pressure on structure of high luminosity stars.

Both $\beta$ Cep and SPB instability domains are very sensitive to the assumed metallicity and shrink with decreasing value of $Z$ (see Dziembowski 1998).

Observational data include results obtained with Hipparcos (Waelkens et al. 1998). Because of limited space, we do not go beyond a simple illustrative comparison. The theoretical instability domains fit almost all of the observed $\beta$ Cephei and the SPB variables.

Acknowledgments. Most of the theoretical results presented here were obtained and discussed in a group headed by Wojciech Dziembowski (WD, Paweł Moskalik, Ryszard Sienkiewicz, AAP). I appreciate a support due to grants KBN 2-P03D-014-14, RFBR 98-02-16734 and a travel grant from IAU. Many thanks to the organizers of the meeting for their hospitality.

\section{References}

Dziembowski W. A. (1977): Oscillations of giants and supergiants. Acta Astron. 27, 95-126

Dziembowski, W. A. (1995): Pulsation in hot stars. Astrophysical applications of powerful new databases, eds. Adelman S.J., Wiese W.L., ASP Conf. Ser., vol. 78, 275-289

Dziembowski, W. A. (1998): B star pulsation - theory and seismological prospects. New eyes to see inside the Sun and stars, Proc. IAU Symp. 185, eds. Deubner F.L., Christensen-Dalsgaard J., Kurtz D.W., Kluwer, Dordrecht, 355-360

Dziembowski W. A., Moskalik P., Pamyatnykh A. A. (1993): The opacity mechanism in B-type stars - II. Excitation of high-order $g$-modes in main-sequence stars. MNRAS 265, 588 600

Garcia J. R., Cebral J. R., Scoccimarro E. R., et al. (1995): A catalogue of variable stars in the lower instability strip. A\&AS 109, 201-262

Gautschy, A., Saio, H. (1995): Stellar pulsations across the HR diagram. I. ARA\&A 33, $75-113$

Gautschy, A., Saio, H. (1996): Stellar pulsations across the HR diagram. II. ARA\&A 34, 551-606

Iglesias, C. A., Rogers, F. J. (1996): Updated OPAL opacities. ApJ 464, 943-953

North P., Paltani S. (1994): HD 37151: a new "slowly pulsating B star". A\&A 288, $155-164$

Pamyatnykh A. A. (1998): Pulsation instability domains in the upper main sequence. A half-century of stellar pulsation interpretations, eds. Bradley P.A., Guzik J.A., ASP Conf. Ser., vol. 135, 268-269

Seaton, M. J. (1996): Interpolations of Rosseland-mean opacities for variable $X$ and $Z$. MNRAS 279, 95-100

Sterken C., Jerzykiewicz M. (1993): $\beta$ Cephei stars from a photometric point of view. Space Sci. Rev. 62, 95-171

Waelkens, C., Aerts C., Kestens E., et al. (1998): Study of an unbiased sample of B stars observed with Hipparcos: the discovery of a large amount of a new slowly pulsating B stars. A\&A 330, 215-221 


\section{Discussion}

J. Cassinelli: The star $\epsilon$ CMa lies just beyond the red edge of the $\beta$ Cep instability zone and has a very large EUV flux (in the $500-700 \AA$ region). Is it possible that pulsational energy that arises deep in the envelope is deposited as heat rather than as an organised pulsation?

A. Pamyatnykh: The red edge of the $\beta$ Cep instability domain is determined in our computations in the same way as the blue edge: if the driving metal opacity bump is too close to the surface (a model to the left of the blue edge), a star will be stable because this potential driving zone will be in thermal equilibrium due to the very short thermal timescale. If the driving metal opacity bump is located too deep in the envelope (a model to the right of the red edge), then: (i) the damping above this region will be activated; (ii) the oscillation amplitude of acoustic-type modes in the driving zone is significantly smaller; and (iii) the oscillations in deep layers are nearly adiabatic. Due to all these factors, the star will be stable as well. So, formally, we do not need any additional energy losses to stabilise oscillations to the right of the red edge. However, the position of the red edge depends quite strongly on stellar parameters, especially on metal abundance and also on the model of the atmosphere, which is important to determine conditions of possible dissipation of pulsational energy in the outermost layers. Therefore, the EUV flux you mentioned may indeed be a manifestation of such an energy dissipation. More definitive answers require a detailed theoretical study of this star and its oscillations.

M. Marlborough: Is it possible in a rapidly rotating B star, e.g., for the polar regions to be stable while the equatorial regions are unstable due to opacity effects?

A. Pamyatnykh: In the evolutionary computations we take the rotational effect into account by adding a correction due to an averaged centrifugal force to the hydrostatic equilibrium equation, so the modified structure of a star remains spherically symmetric. Therefore, there is no difference in the driving effect between polar and equatorial regions in our models. Lee (1998, ApJ 497, 312) found that due to rotational deformation of the outer envelope the damping effect above the main driving metal-bump region can become significant in the equatorial zone. It may result in the stabilisation of some axisymmetric non-radial modes of a rapidly rotating $B$ star which are unstable in the non-rotating case.

A. Maeder: You have shown the metal opacity peak at $200000 \mathrm{~K}$, but in your plots of $\log \kappa$ vs. $\log T$ and $\log P$ there was another peak at higher temperatures. Is it driving any pulsations?

A. Pamyatnykh: The deeper opacity bump near $\log T \sim 6.3-6.4$ is produced by fine-structure transitions in iron-group elements as well as in carbon and oxygen. It may be important to helioseismology and to the lithium 
problem in low mass stars due to some modification of solar and stellar interior structure (see, e.g., the review by Rogers \& Iglesias on astrophysical opacity, Science, 1994, 263, 50). As for the driving of pulsations: this bump may be potentially important for some very hot stars to the left of the upper main sequence, but I don't know any study about this matter.

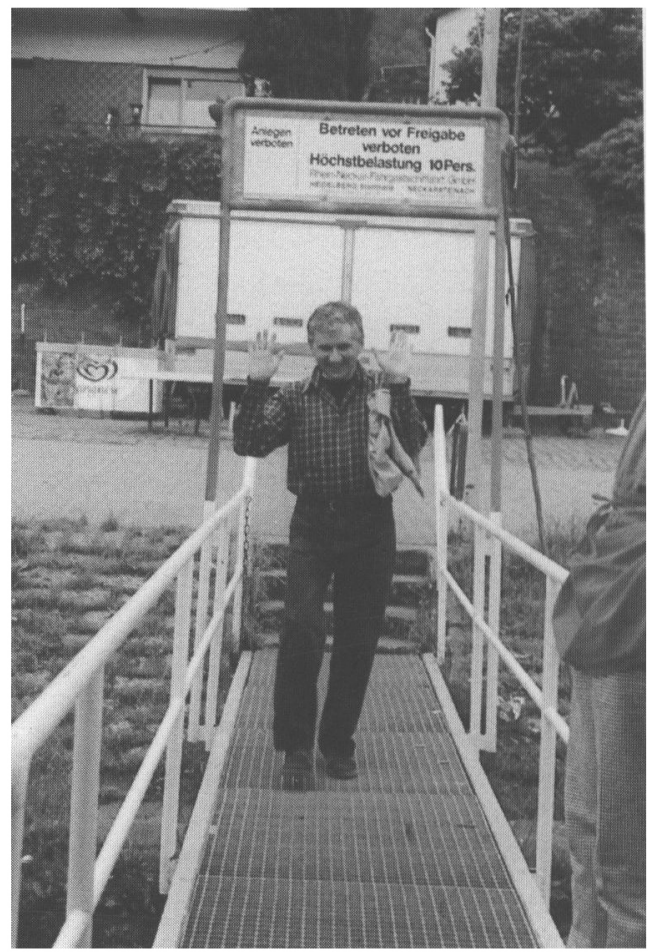

Alosza Pamyatnykh (returns from being lost in the Odenwald) 